\title{
Design on Open-Loop Bandpass Filter with Multiple Harmonics Suppression
}

\author{
Liu He ${ }^{\mathrm{a}}$, Xing-Jian Zhong ${ }^{\mathrm{a}}$, De-Xin $\mathrm{Qu}^{\mathrm{a}}$, Zhen-Dong Fan ${ }^{\mathrm{a}}$ and Kai Shi ${ }^{\mathrm{b}}$ \\ ${ }^{a}$ College of Communications Engineering, The Army Engineering University of PLA, Nanjing 210007, China \\ ${ }^{b} 31121$ PLA Troops, Nanjing 210005, China
}

Received: 25 April 2018; Accepted: 19 July 2018; Published: 08 September 2018

\begin{abstract}
In this paper, a simple method based on coupling control theory to suppress multiple harmonics in open-loop bandpass filters is proposed. Coupled feeding is utilizing in this paper, and by choosing proper coupling length and position of the input/output port, multiple harmonics could be suppressed. The input impedance of microstrip line with its end open-circuited is firstly showed and the theory on harmonics suppression is then analyzed to choose a proper coupling position. A second-order open-loop bandpass filter is designed and manufactured, measured and manufactured results both have a stopband to $5 f_{0}$ with rejection more than $20 \mathrm{~dB}$. On the base of the filter proposed, the stopband can be broaden to $10 f_{0}$ by adding two stubs. It well validates the proposed solution.
\end{abstract}

Index Terms: Harmonic suppression,bandpass filter, coupling structure, open-loop resonator.

(C) 2018 Published by MECS Publisher. Selection and/or peer review under responsibility of the Research Association of Modern Education and Computer Science

\section{Introduction}

Microstrip bandpass filters are widely used in the front-end of ratio frequency (RF) communication, of which the open-loop bandpass filter is a popular design for its planar structure, narrow bandwidth and easy fabrication process. The rejection level in the upper stopband of this type of filter, however, is often degraded by the spurious responses at multiples of the designed frequency, undesired harmonics would seriously degrade the performance of whole RF system due to its invalidation of the noise in the spurious passband.

Several solutions have been proposed to tackle this problem [1-11]. The simplest way is cascading element with the property of low-pass or bandstop, but this will increase the insertion loss in the passband and the overall circuit size. In $[1,2]$, spur-line is utilized in inner microstrip line without any penalty of increasing size. To

* Corresponding author : Liu He;

E-mail address:15651766397@163.com 
equalize the velocities of the even- and odd-mode, over-coupled end stages are applied in parallel-coupled microstrip filters to suppress the spurious passband at $2 f_{0}$ [3]. The stepped-impedance resonators (SIRs) could be utilized to shift the second harmonic to higher frequency beyond $2 f_{0}$ [4-6], lager the ratio of impedance is, higher the second resonant frequency is. Besides, different SIRs with the same designed frequency but staggered higher resonant frequency are used to design filters and a wider stopband could be achieved. To eliminate spurious harmonics, continuously perturbing the width of the coupled lines is also used. In [7,8], a microstrip wiggly-line filter is demonstrated by use of sinusoidal rule. Moreover, other special structures are also studied such as overlay dielectric [9], electromagnetic band-gap (EBG) [10], lumped capacitors[11] and ground-plane aperture (DGS) $[12,13]$. In [14], a miniature sized microstrip UWB (ultra wideband) BPF (bandpass filter) having highly attenuated upper stopband performance using a dual MMR (multimode resonator) and the DGS (defected ground structure) is proposed. Besides, cross-coupling [15] and split-ring resonators[16] are also adopted in the application of suppressing spurious responses.

In [17], a novel method to utilize input impedance of input/output microstrip line is proposed. They present a filter with $\lambda / 4$ input microstrip line replaced by $\lambda / 2$ microstrip line, the spurious passband at $2 f_{0}$ is suppressed and the selectivity is promoted. This method shows its advantage over other methods, it has little change on original structure of bandpass filter, only by adjusting the length and position of the I/O port, the harmonic could be rejected with no influence on the passband.

This paper presents some promoted methods based upon previous theory in [19], and similarly by adjusting the position of the I/O port coupling to the resonators, more harmonics could be suppressed. In previous work, only the input/output transmission line is utilized for suppressing the harmonics. However, in fact, the open-loop resonator is also microstrip line with length of $\lambda / 2$ which means that there exist extra transmission zeros by proper design. In this paper, both $\mathrm{I} / \mathrm{O}$ transmission line and the open-loop resonators are taken use of, we analyze input impedance of $\lambda / 2$ microstrip line and more harmonics are suppressed only by adjusting the coupling position. For a better, two open-stubs are utilized and the design shows a stopband beyond $10 f_{0}$.

\section{Theory}

As we all know, the impedance is varied with the position of microstrip line and frequency, and so is with current density, if the low current density region is applied at harmonics, coupling energy would be zero and spurious resonance could be suppressed. Based upon this, the distribution of input impedance could be firstly analyzed, microstrip line with its end open-circuited is considered. Just as informed in [18], the input impedance is as follow:

$$
Z_{\text {in }}=-j z_{0} \cot \beta_{0} l
$$

where $\beta_{0}$ is the phase constant, and $Z_{0}$ refers its characteristic impedance. Because both the I/O port and resonators are constituted of $\lambda / 2$ microstrip line, so in the following text, only half wavelength at designed frequency is concerned.

To show the theory of adjusting coupling position to suppress spurious passband, the input impedance at spurious resonances could be also showed as

$$
Z_{\text {in }}=-j z_{0} \cot n \beta_{0} l
$$

where $n$ refers to the upper order of the spurious resonance. It could be easily seen that the function is periodical and repetitive based upon on that of the designed frequency. As we all know, a null impedance value means a maximum current density and maximum coupling strength, on the contrary, a enormous value of impedance means a minimum current density and weak coupling strength in the ends of the line or the 
resonator. Supposing that at some position of the $\lambda / 2$ microstrip line, the minimum current density appeared at harmonics, but with a proper value at the designed frequency, the harmonics could be suppressed. And according to the corresponding relationship of current density and input impedance, what we should focus on is the minimum impedance at $f_{0}$ and the maximum values at higher order harmonics.

From Fig.1.(a), at the middle of the microstrip line, the input impedance is the lowest and about zero, while at the both ends, it is the largest. So when the frequency is at $f_{0}$, what we should focus on is the middle of half wave-length microstrip line to make sure enough coupling energy. But the status is different when the frequency shift to $2 f_{0}$ which is the first spurious frequency point.

From Fig.1(b), the null impedance value occurs at 3/4 and 1/4 wavelength location of the open-circuited, while high impedance occurs at both ends and in the middle of the line. Based upon the theory of coupling strength above, the spurious resonance should be suppressed, and the high impedance region should be applied in design. Just the same as the situation when the frequency is at $2 f_{0}$, the functional image of input impedance at $3 f_{0}$ is expanded periodically. At $1 / 6,1 / 2$, and $5 / 6$ of the entire microstrip line, low impedance occurs, while at the both ends, $1 / 3$, and $2 / 3$ of the line, high impedance occurs. Similarly, the high impedance region at $3 f_{0}$ should be applied to our design. It is then expected that while the resonators are set and coupled to the maximum coupling strength of the microstrip lines, the resonant mode can be passed and created. Otherwise, while the resonators are set and coupled to the low coupling strength of the microstrip lines, the resonant mode might be suppressed.

In [17], only the spurious resonance at $2 f_{0}$ is considered. In this design, deep study on the structure of open-loop bandpass filter is made. In a open-loop resonator bandpass filter, there exist four coupling structures, and a lot of experiments show that the coupling structure should be symmetric to suppress harmonics. So in this paper, the coupling control with the resonator coupling to the $\mathrm{I} / \mathrm{O}$ microstrip line is used to suppress the spurious resonance at $2 f_{0}$, while the coupling control with one resonator coupling to another resonator is utilized for suppression of harmonics at $3 f_{0}$.

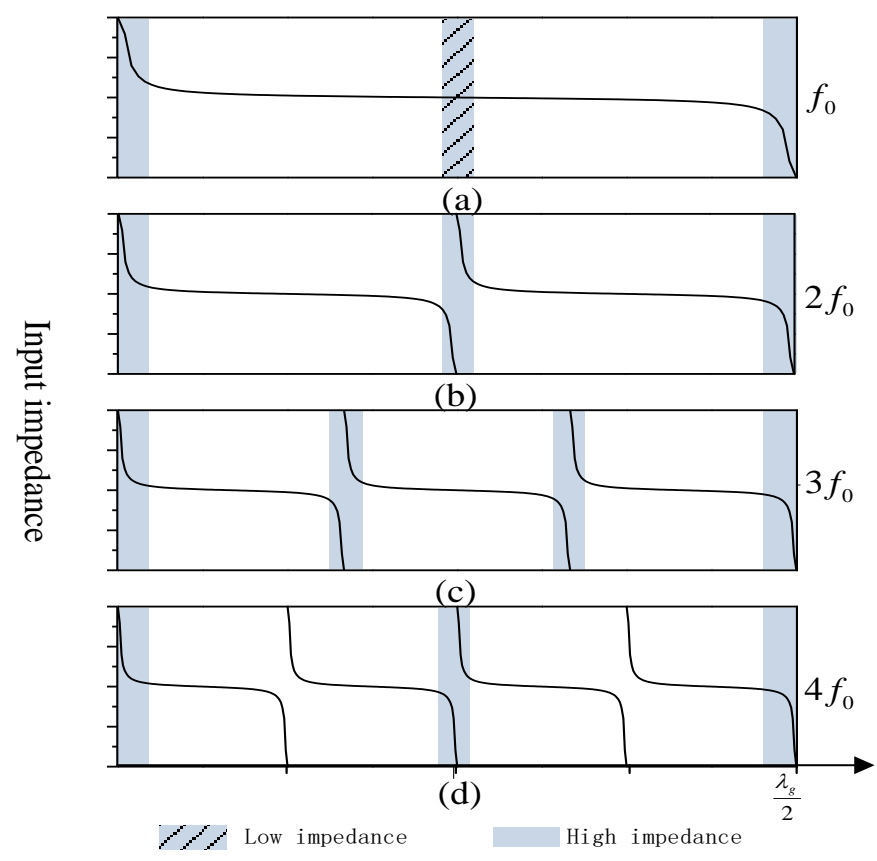

Fig.1. Input impedance with the location of the microstrip line and the frequency altered, (a) the frequency is at $f_{0}$, (b) the frequency is at $2 f_{0}$, (c) the frequency is at $3 f_{0}$, (d) the frequency is at $4 f_{0}$. 


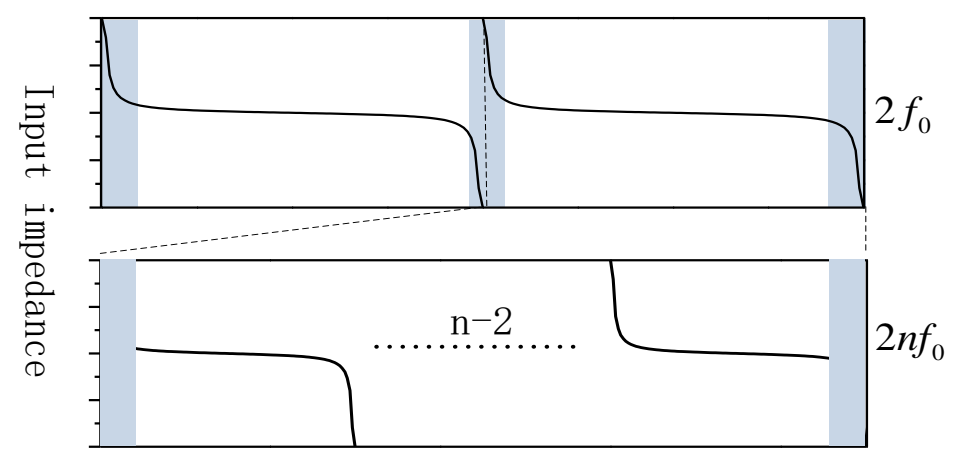

Fig.2. Input impedance with the location of the microstrip line and the frequency altered , (a) the frequency is at $2 f_{0}$, (b) the frequency is at $2 \mathrm{n} f_{0}$, where $\mathrm{n}$ is a natural number and $\mathrm{n} \geq 0$.

More study on the coupling control is made in this paper, from Fig.1(a) and Fig.1(d), some laws are showed preliminarily. Fig. 2 shows more details about the laws. According to the periodicity and the repeatability of the function image at harmonics, when at some location of $m f_{0}$, there exists high impedance, and at the same position, there also exists high impedance at $n \times m f_{0}$. It means that if the spurious resonance at $m f_{0}$ is suppressed, the harmonics at $n \times m f_{0}$ could be suppressed at the same time. So the structure above could suppress the harmonics at $2 f_{0}, 3 f_{0}, 4 f_{0}, 6 f_{0}, 8 f_{0}, 9 f_{0}$ and $10 f_{0}$. Only the spurious resonances at $5 f_{0}$ and $7 f_{0}$ exist. In this paper, to get better performance, two stubs to suppress the harmonics at $5 f_{0}$ and $7 f_{0}$ are adopted and the stopband could be pushed to $11 f_{0}$.

\section{Filter Design}

To validate the theory above, use ADS to design a second-order open-loop bandpass filter which centers at $2.5 \mathrm{GHz}$ with a fractional passband 5\%. All design is based upon the substrate Rogers5880 with $0.017 \mathrm{~mm}$ thick copper, 2.2 dielectric constant 0.0009 dielectric loss tangent and the thickness of the substrate is $0.508 \mathrm{~mm}$.

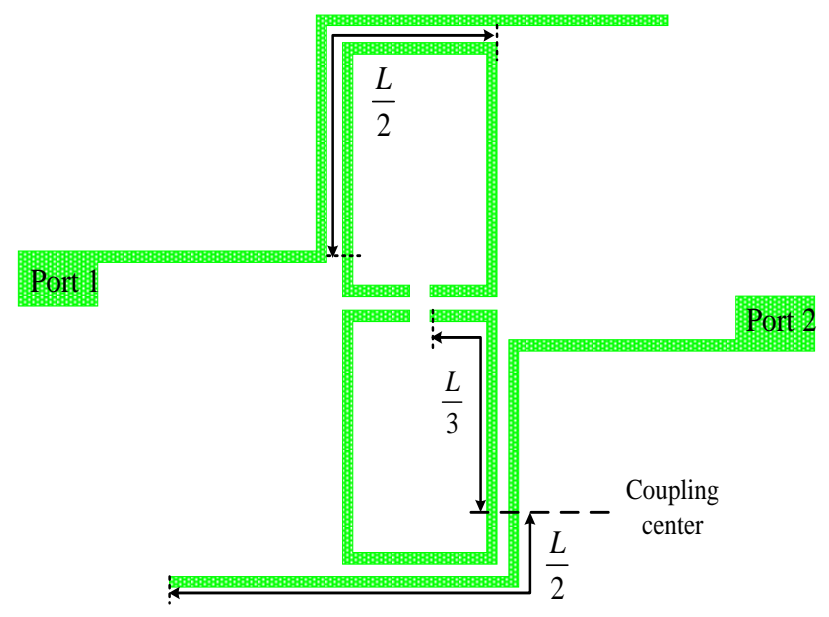

Fig.3. Structure of the designed open-loop bandpass filter. 
Fig. 3 shows the designed structure. The middle of the I/O coupling feeding line align at the 1/3 $\lambda$ point of the open-loop resonator in order to suppress the harmonics at $3 f_{0}$. For the I/O microstrip line, the middle point is where coupling strength is the largest at the designed frequency but null coupling when the frequency is at $2 f_{0}$, this could realize the suppression of the first harmonic. While for the resonator, $1 / 3$ or $2 / 3$ wavelength is chosen as the coupling point, though the coupling strength is not the largest, it can meet the demands when the frequency is at $3 f_{0}$.

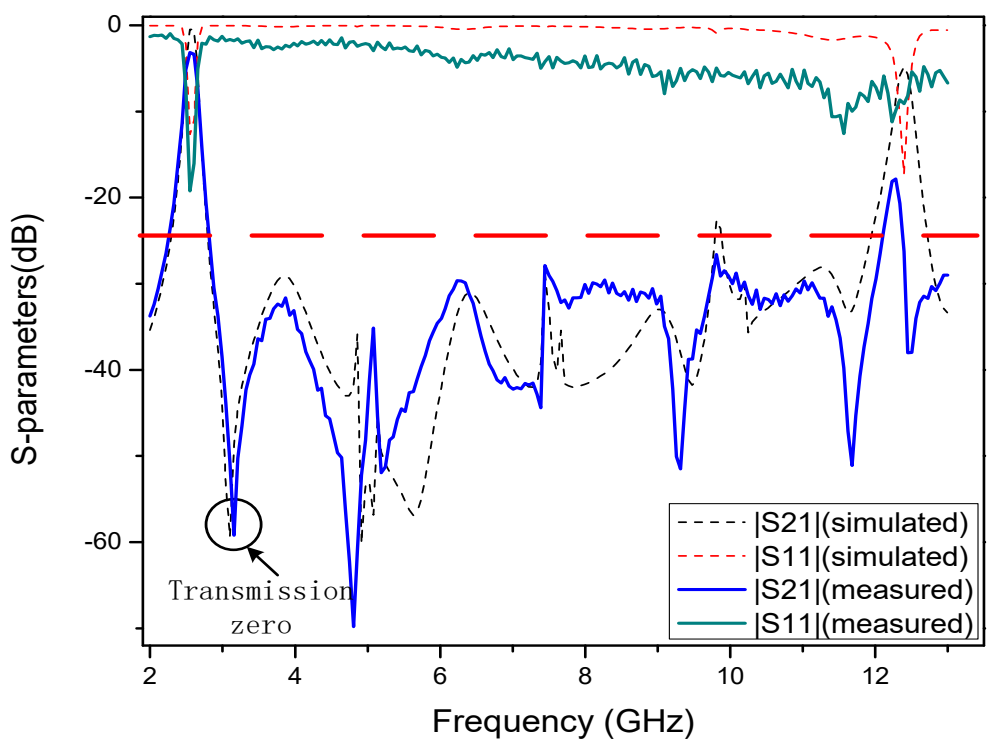

Fig.4. Measured and simulated results of the designed open-loop bandpass filter.

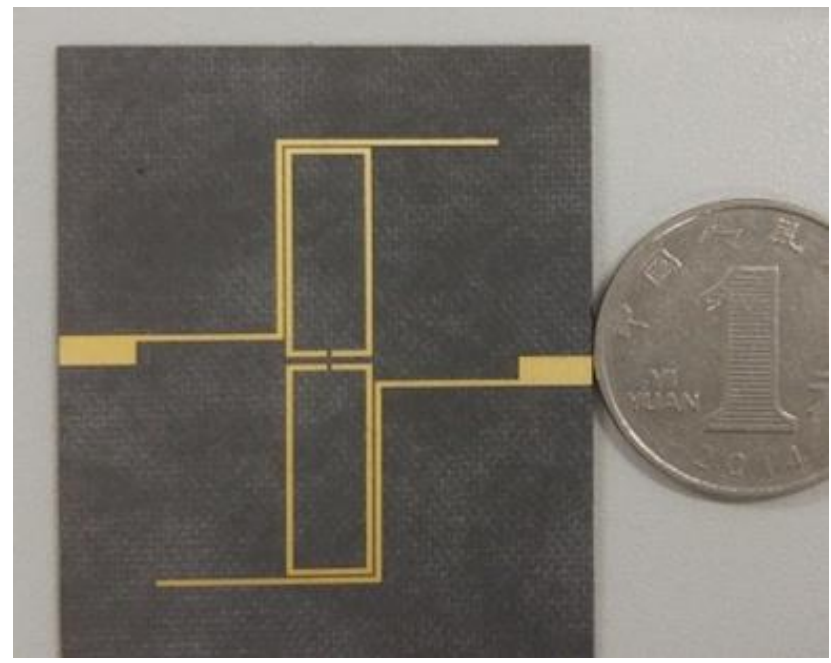

Fig.5. Photo of the manufactured filter. 
The filter is manufractured as in Fig.5 and the simulated and measured results is shown in Fig.4. As is shown in Fig.4, the center frequency is about $2.5 \mathrm{GHz}$. The insertion loss of the measured result is $3.1 \mathrm{~dB}$ and is $1.6 \mathrm{~dB}$ of the simulated result, the difference is mainly caused by the measuring error and manufacturing error. It can be seen that S11 of the measured result is zigzag but not straight, which is mainly caused by the error of the measuring instrument. In the stopband, a transmission zero is introduced at adjacent passband about $3.1 \mathrm{GHz}$, and the harmonics here could be suppressed about $60 \mathrm{~dB}$ which improves the selectivity of the filter. The harmonics at $2 f_{0}$ could be suppressed about $50 \mathrm{~dB}$, the harmonics at $3 f_{0}$ could be suppressed about $45 \mathrm{~dB}$, and the harmonics at $4 f_{0}$ could be suppressed about $30 \mathrm{~dB}$. On the whole, at the frequency from $3 \mathrm{GHz}$ to $12 \mathrm{GHz}$, the harmonics could be suppressed beyond $20 \mathrm{~dB}$, which consistent well with the theoretical results.

\section{Improved Design}

According to the theory above, this method can suppress more harmonics. In the design above, using coupling feeding at $\mathrm{L} / 3$ of the resonator can suppress the harmonics at $6 f_{0}$ and $9 f_{0}$. Using coupling feeding at $\mathrm{L} / 2$ of the I/O microstrip line can suppress the harmonics at $6 f_{0}, 8 f_{0}$ and $10 f_{0}$. If we add two $\lambda / 4$ open-stubs to suppress the harmonics at $5 f_{0}$ and $7 f_{0}$, the stopband would be easily broaden to $11 f_{0}$. On the base of the structure as in Fig.3., two stubs is added as in Fig.6, the width of the stubs is the same as the resonator.

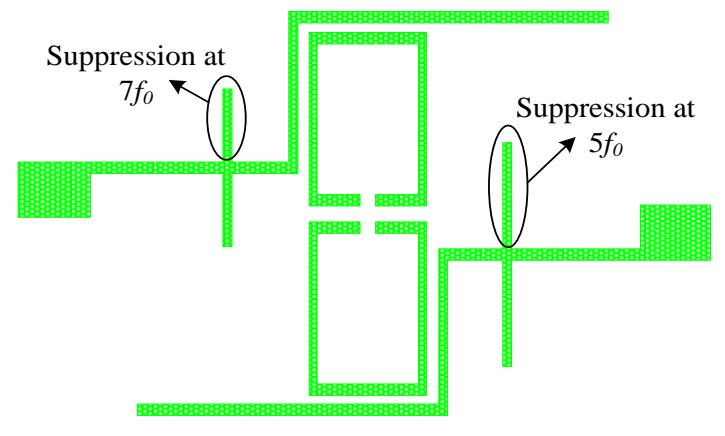

Fig.6. Improved design of the open-loop bandpass filter.

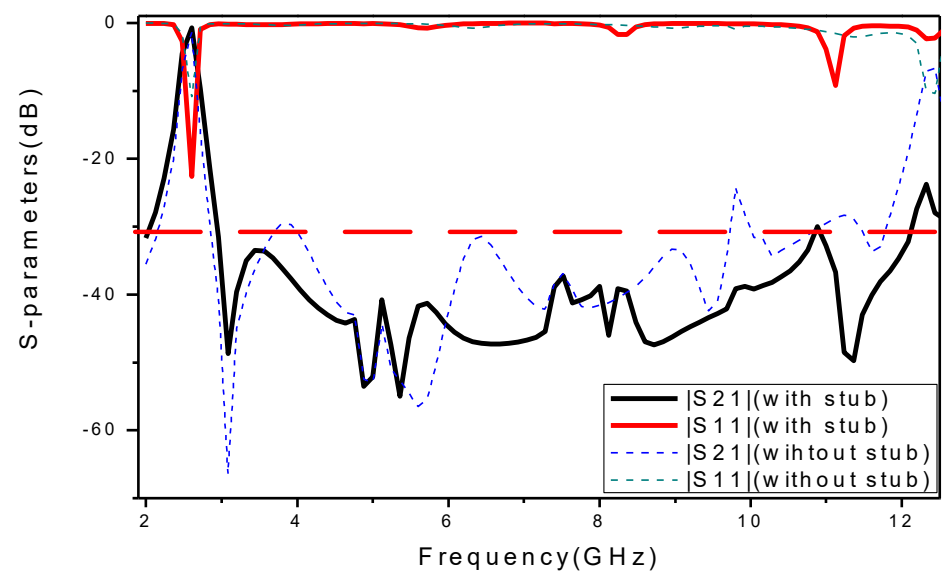

Fig.7. Simulated results of the open-loop bandpass filter with stubs and without stubs, the frequency is from $2 \mathrm{GHz}$ to $12.5 \mathrm{GHz}$. 


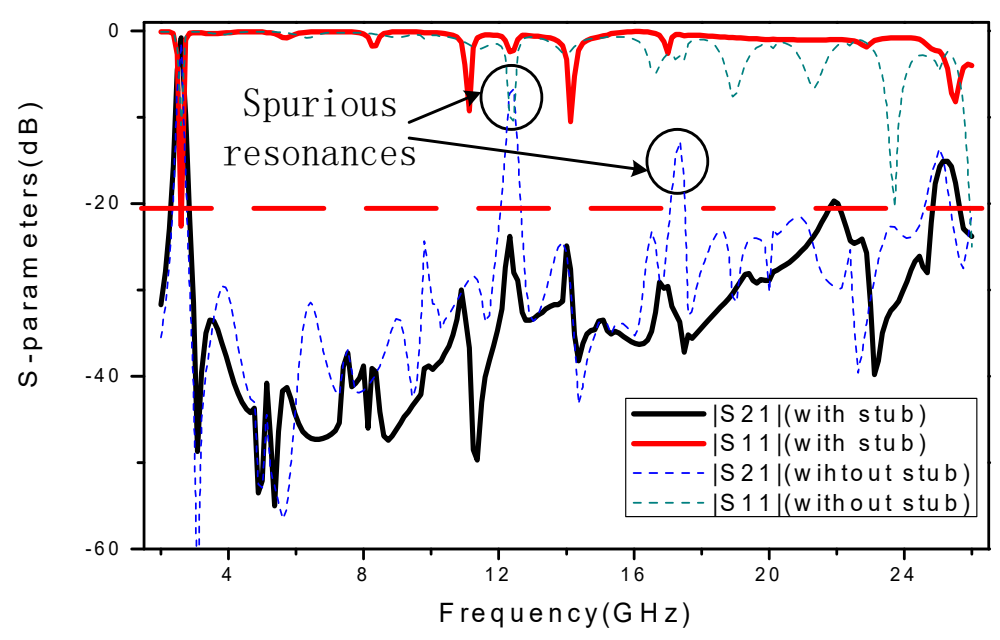

Fig.8. Simulated results of the open-loop bandpass filter with stubs and without stubs, the frequency is from $2 \mathrm{GHz}$ to $26 \mathrm{GHz}$.

In Fig.6, two stubs are introduced to the filter, and by ADS simulating, the best position to add them is decided. Besides, in order to clearly see the difference and the influence brought by the two stubs, other parameters of the structure was little changed. The simulated results of the filter are shown in Fig.7 and Fig.8. Fig. 7 focuses on the frequency from $2 \mathrm{GHz}$ to $12.5 \mathrm{GHz}$ in order to more clearly see the difference near the passband. The performance of the passband hardly changes, the rejection level of the transmission zero at $3.1 \mathrm{GHz}$ gets worse after adding two stubs. However, the rejection level at $4 \mathrm{GHz}, 6.5 \mathrm{GHz}, 9 \mathrm{GHz}$ and $10 \mathrm{GHz}$ definitely gets better in the improved filter. On the whole, at the frequency from $3 \mathrm{GHz}$ to $12 \mathrm{GHz}$, the harmonics could be suppressed $30 \mathrm{~dB}$.

Fig. 8 focuses on the frequency far away from the passband. Before adding the stubs, there are two serious spurious resonances at $5 f_{0}$ and $7 f_{0}$. After adding two $\lambda / 4$ open-stubs, the harmonics have been obviously suppressed more than $20 \mathrm{~dB}$. The insertion loss at the frequency from $18 \mathrm{GHz}$ to $26 \mathrm{GHz}$ is obviously reduced after the structure improved. It well validates the proposed improved design.

\section{Conclusions}

A novel open-loop bandpass filter based on coupling control theory with multiple harmonics suppression is proposed in this paper. Coupled feeding is utilizing in this paper, and only by choosing proper coupling length and position of the input/output port, multiple harmonics could be suppressed. The input impedance of microstrip line with its end open-circuited is showed and how to suppress harmonics is analyzed to choose a proper coupling position. A second-order open-loop bandpass filter is first designed and manufactured. The center frequency is about $2.5 \mathrm{GHz}$, the insertion loss of the measured result is $3.1 \mathrm{~dB}$ and is $1.6 \mathrm{~dB}$ of the simulated result, $\mathrm{S} 11$ of the measured result is zigzag but not straight. These are mainly caused by the joint loss and measuring error. Measured and manufactured results both have a stopband to $5 f_{0}$ with rejection more than $20 \mathrm{~dB}$. On the basis of the filter proposed, two $\lambda / 4$ open-stubs are added to suppress the harmonics at $5 f_{0}$ and $7 f_{0}$. As a result, the stopband could be broaden to $10 f_{0}$ and the insertion loss is obviously reduced far away from the passband. The design example shows good consistency with the theory and the measurements results agree well with the simulation results. 


\section{Acknowledgements}

This work was supported by the National Natural Science Foundation of China (NSFC) under Grant 61271105 and Grant 6127110.

\section{Reference}

[1] W. H. Tu and K. Chang, Compact microstrip bandstop filter using open stub and spurline, IEEE Microw. Wireless Compon. Lett., vol. 15, no. 4, pp. 268-270, Apr. 2005.

[2] Ching- Luh Hsu and Jen- Tsai Kuo, A two- Stage SIR Bandpass Filter With an Ultra-Wide Upper Rejection Band, IEEE Microwave and Wireless Components papers, Vol. 17, Issue: 1, pp. 34- 36, Jan. 2007.

[3] J. T. Kuo, S. P. Chen, and M. Jiang, Parallel-coupled microstrip filters with over-coupled end stages for suppression of spurious responses, IEEE Microw. Wireless Compon. Lett., vol. 13, no. 10, pp. 440-442, Oct. 2003.

[4] M. Makimoto and S. Yamashita, Bandpass filters using parallel coupled stripline stepped impedance resonators, IEEE Trans. Microw. Theory Tech., vol. MTT-28, no. 12, pp. 1413-1417, Dec. 1980.

[5] J. T. Kuo, W. H. Hsu, and W. T. Huang, Parallel coupled microstrip filters with suppression of harmonic response, IEEE Microw.Wireless Compon. Lett., vol. 12, no. 10, pp. 383-385, Oct. 2002.

[6] J. T. Kuo and E. Shih, Microstrip stepped impedance resonator bandpass filter with an extended optimal rejection bandwidth, IEEE Trans. Microw. Theory Tech., vol. 51, no. 5, pp. 1554-1559, May. 2003.

[7] T. Lopetegi, M. A. G. Laso, J. Hernández, M. Bacaicoa, D. Benito, M. J. Garde, M. Sorolla, and M. Guglielmi, New microstrip wigglyline filters with spurious passband suppression, IEEE Trans. Microw. Theory Tech., vol. 49, no. 9, pp. 1593-1598, Sept. 2001.

[8] T. Lopetegi, M. A. G. Laso, F. Falcone, F. Martin, J. Bonache, J. Garcia, L. Perez-Cuevas, M. Sorolla, and M. Guglielmi, Microstrip 'wiggly-line'bandpass filters with multispurious rejection, IEEE Microw. Wireless Compon. Lett., vol. 14, no. 11, pp. 531-533, Nov. 2004.

[9] J. -T. Kuo; Meshon Jiang, Enhanced microstrip filter design with a uniform dielectric overlay for suppressing the second harmonic response, IEEE Microwave and Wireless Components papers, Vol.14, Issue: 9, pp. 419 - 421, DOI: 10.1109/LMWC.2004. 832068.

[10] W.M. Zhang, J.F. Mao, and X.W. Sun, Compact resonant bandpass filter based on PBG structure, Electronics papers, vol. 39, no. 3, pp. 615-617, Apr. 2003.

[11] Zheng Chen, Liu He, De-Xin Qu, Xing-Jian Zhong, Microstrip dual-band bandpass filter fed with Lumped capacitors, I. J. Wireless and Microwave Technologies, pp. 13-17, DOI: 10.5815/ijwmt. 2017. 06. 02

[12] Vidhya K, Jayanthy T, Performance enhancement of microstrip hairpin bandpass filter using dumbbell DGS and split ring resonator DGS, International Journal of Information and Electronics Engineering, Vol. 1, No. 1, July 2011.

[13] Amar Touhami N, Zakriti A, Lamsalli M, Boussouis M, Tribak A, Spurious response suppression in three poles hairpin bandpass filter using defected ground structures, 1st Annual International Interdisciplinary Conference, AIIC 2013, 24-26 April, Azores, Portugal.

[14] Aditi Senguptaa, Somdotta Roy Choudhuryb, Santanu Das, Design of an UWB bandpass filter using dual MMR with highly attenuated upper stopband using DGS", I.J. Wireless and Microwave Technologies, PP. 58-69, DOI: 10. 5815/ijwmt. Jun. 2018.

[15] Jhe-Ching Lu; Chi-Yang Chang, Generalized chebyshev response parallel-coupled filter with suppression of $2 \mathrm{f0}$ spurious passband, Asia-Pacific Microwave Conference, 2008, pp. 1 - 4, DOI: 10.1109. 
[16] Miguel Duran-Sindreu, Adolfo Velez, Francisco Aznar, Gerard Siso, Jordi Bonache, Applications of open split ring resonators and open complementary split ring resonators to the synthesis of artificial transmission lines and microwave passive components, IEEE Trans. Microw. Theory Tech., Vol. 57, Issue: 12, pp. 3395-3403, DOI: 10. 1109/TMTT. 2009. 2033867.

[17] RY Yang, JS Lin, HS Li, A novel coupling method to design a microstrip bandpass filter with a wide rejection band, Progress in Electromagnetics Research C, 2010, 14:45-52.

[18] D. M. Pozar, Microwave Engineering, 3nd edition. New York: Wiley, 2006.

\section{Authors' Profiles}
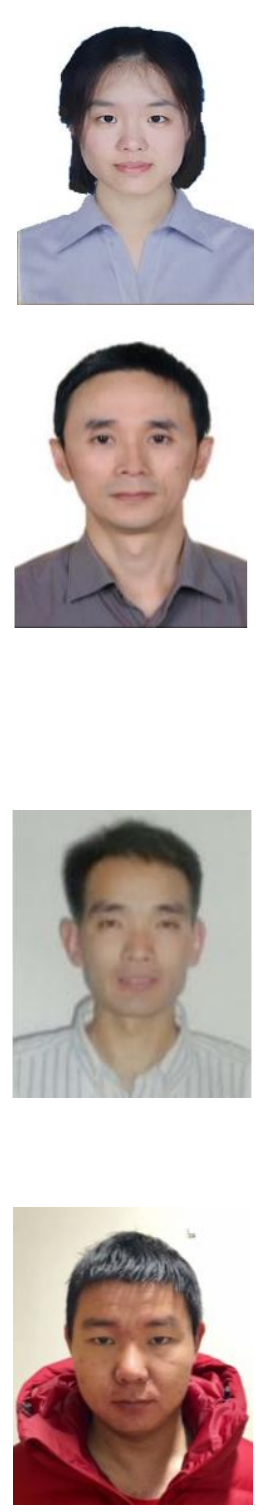

Liu He (born May 4, 1994) is currently working toward the Master degree in electromagnetic field and microwave technology, The Army Engineering University of PLA, Nanjing, Jiangsu, China.

Her current reaserch interests include microwave passive components.

Xing-Jian Zhong received the Ph.D. degree in electromagnetic field and microwave technology from Southeast University, Nanjing, China, in 2014. He is currently a lecturer with The Army Engineering University of PLA, Nanjing, China.

His current research interests include microwave and millimeter-wave (mm-wave) theories and technologies, $\mathrm{RF} /$ microwave components and systems, antennas design.

De-Xin Qu received bachelor degree in radio electronics from Beijing University, Beijing, China, 1987, recieved Master and PH.D. degree in microwave communication from PLA Institute of Communications Engineering, Nanjing, China, 1990 and 1996. He is now a professor with Nanjing University of Posts and Telecommunications, Nanjing, China.

His current research interests include RF/microwave components and systems, Signal Processing.

Zhen-Dong Fan was born in Anhui province, China, 1987. He received bachelor degree in microelectronics from Nanjing University of Posts and Telecommunications, Nanjing, China, 2008. He is an army engineer and currently working toward the Master degree in integrated information business network field, The Army Engineering University of PLA, Nanjing, Jiangsu, China.

His current reaserch interests include microwave passive components. 


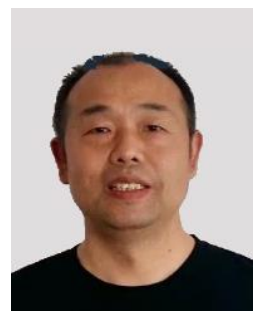

Kai Shi was born in Anhui province, China, 1970. He received bachelor degree in communication engineering from PLA University of Science and Technology, Nanjing, China, 1992. He is now an army engineer in 31121 PLA troops, Nanjing, China.

His current research interests include microwave and millimeter-wave (mm-wave) theories and technologies, RF/microwave components and systems.

How to cite this paper: Liu He, Xing-Jian Zhong, De-Xin Qu, Zhen-Dong Fan, Kai Shi, " Design on Open-Loop Bandpass Filter with Multiple Harmonics Suppression ", International Journal of Wireless and Microwave Technologies(IJWMT), Vol.8, No.5, pp. 27-36, 2018.DOI: 10.5815/ijwmt.2018.05.04 